Fixed Point Theory, 19(2018), No. 1, 167-178

DOI 10.24193/fpt-ro.2018.1.12

http://www.math.ubbcluj.ro/ ${ }^{\text {nodeacj/sfptcj.html }}$

\title{
PROJECTIONS ONTO CONES IN BANACH SPACES
}

\author{
A. DOMOKOS* AND M.M. MARSH** \\ *Department of Mathematics and Statistics \\ California State University at Sacramento \\ 6000 J Street, Sacramento, CA, 95819, USA \\ E-mail: domokos@csus.edu \\ ** Department of Mathematics and Statistics \\ California State University at Sacramento \\ 6000 J Street, Sacramento, CA, 95819, USA \\ E-mail: mmarsh@csus.edu
}

Abstract. We propose to find algebraic characterizations of the metric projections onto closed, convex cones in reflexive, locally uniformly convex Banach spaces with locally uniformly convex dual.

Key Words and Phrases: Banach space, closed convex cone, metric projection.

2010 Mathematics Subject Classification: 41A65, 46C50, 52A27.

Acknowledgment. The authors would like to thank the referee for his/her comments, which helped improve the presentation of the results in the paper.

\section{REFERENCES}

[1] K. Deimling, Nonlinear Functional Analysis, Springer-Verlag Berlin, Heidelberg, New York, Tokyo, 1985.

[2] F. Deutch, Existence of best approximations, J. Approx. Theory, 28(1980), 132-154.

[3] J. Diestel, Geometry of Banach Spaces - Selected Topics, Lecture Notes in Math., vol. 485, Springer-Verlag, Berlin, Heidelberg, New-York, 1975.

[4] S.S. Dragomir, Semi-Inner Products and Applications, Nova Science Publishers, Inc., Hauppauge, NY, 2004.

[5] S. Fitzpatrick, R.R. Phelps, Differentiability of the metric projection in Hilbert spaces, Trans. Amer. Math. Soc., 270(1982), 483-501.

[6] J.R. Giles, Classes of semi-inner product spaces, Trans. Amer. Math. Soc., 129(1967), 436-446.

[7] J.M. Ingram, M.M. Marsh, Projections onto convex cones in Hilbert spaces, J. Approx. Theory, 64(1991), no. 3, 343-350.

[8] R.C. James, Orthogonality and linear functionals in normed linear spaces, Trans. Amer. Math. Soc., 61(1947), 265-292.

[9] A.G. Kartsatos, A note on the duality mapping of a locally uniformly Banach space, Nonlinear Anal., 71(2009), 5509-5512.

[10] R. Larsen, Functional Analysis, Marcel Dekker, 1973.

[11] G. Lumer, Semi-inner product spaces, Trans. Amer. Math. Soc., 100(1961), 29-43.

[12] S.Z. Németh, A duality between the metric projection onto a convex cone and the metric projection onto its dual in Hilbert spaces, Nonlinear Anal., 97(2014), 1-3. 
[13] A.B. Németh, S.Z. Németh, Lattice-like operations and isotone projection sets, Linear Algebra Appl., 439(2013), 2815-2828.

[14] J.-P. Penot, R. Ratsimahalo, Characterizations of metric projections in Banach spaces and applications, Abstr. Appl. Anal., 3(1998), no. 1-2, 85-103.

[15] I. Singer, Best Approximation in Normed Linear Spaces by Elements of Linear Subspaces, Springer-Verlag Berlin, Heidelberg, 1970.

[16] I. Singer, The Theory of Best Approximation and Functional Analysis, Conference Board of the Mathematical Sciences Regional Conference Series in Applied Mathematics, \# 13. Society for Industrial and Applied Mathematics, Philadelphia, Pa., 1974.

[17] Z. Xiyin, The normalized duality mappings in Banach spaces, Nonlinear Anal., 24 (1995), 989995.

Received: May 25, 2016; Accepted: August 12, 2016. 
